\title{
Design of magnetorheological fluid dynamometer which electric current and resisting moment have corresponding relationship
}

\author{
Luo Yiping, Xu Biao, Ren Hongjuan, Chen Fuzhi \\ College of Automobile Engineering, Shanghai University of Engineering Science, Shanghai, China \\ Email address: \\ lyp777@sina.com (Luo Yiping), xubiao0813@163.com (Xu Biao), ren-hongjuan@163.com (Ren Hongjuan), \\ 284774881@qq.com (Chen Fuzhi)
}

\section{To cite this article:}

Luo Yiping, Xu Biao, Ren Hongjuan, Chen Fuzhi. Design of Magnetorheological Fluid Dynamometer which Electric Current and Resisting Moment have Corresponding Relationship. Automation, Control and Intelligent Systems. Vol. 2, No. 2, 2014, pp. 16-20. doi: 10.11648/j.acis.20140202.11

\begin{abstract}
Based on the research of the new material magnetorheological fluid, magnetorheological fluid dynamometer is designed. Under the premise of certain structure size and material, there is a one-to-one correspondence between MRF dynamometer loading current and load torque provided with the machine electricity and the theoretical calculation. This papergives the design method and specific geometric parameters of magnetorheological fluid dynamometer. The process of magnetorheological fluid dynamometer theory design is obtained by taking a specific model motor as an example, which provides a theoretical basis for the application of MRF in the field of dynamometer.
\end{abstract}

Keywords: MRF, Dynamometer, Load Torque, Design, Corresponding Relationship

\section{Introduction}

Magnetorheological Fluid, called MFR, is an active branch in the research of intelligent material field. MFR is made of tiny soft magnetic particles and non-conducting magnetic mother liquid with high permeability and low hysteresis of magnetic and mixed with an emulsifier[1]. Under the action of external magnetic field, MRF can instantly ( in milliseconds ) achieve consecutive reversible transition between the low viscosity easy flow of Newtonian fluid and high viscosity hard plastic Bingham[2]. It has a wide range of applications in many fields because of its "liquid", "solid" state transition reversible, controllable and rapid and other outstanding technological features[3]. In the current technology, Bossis and Cutillas et al from French University of Nice has done a lot of work in the MRF mechanism research, especially in the aspect of microstructure analysis[4]. Kormann et al from Germany BASF $G$ have developed a stable of nanoscale MRF[5]. The engineering and technical personnel Lord company has developed a vehicle seat suspension damper[6]. Gm Foister and Gopalswamy developed magnetorheological Fluid and magnetorheological clutch[7]. Jianhua $\mathrm{Ni}[8]$ from Xi'an Jiaotong University had carried out a research on the application of magnetorheological damper in the vehicle suspension control.

The existing measurement devices for rotating parts (engines, motors, etc. ) power are hydraulic dynamometers, electricity dynamometer, eddy current dynamometer, etc. which can relatively accurately measure the power of the rotary member to a certain extent but also have such defects as large size, slow response, energy consumption, expensive. Magnetorheological fluid power measuring device has broad prospects with its compact structure, rapid response and low power consumption.

\section{Principle of Work}

Rotating parts power calculated formula is:

$$
P=\frac{n T}{9550}
$$

Where $\mathrm{P}$ is the power of rotating parts $(\mathrm{kW}), \mathrm{n}$ is the rotation speed $\left(r \cdot \mathrm{min}^{-1}\right), \mathrm{T}$ is the torque $(\mathrm{N} \cdot \mathrm{m})$.

Magnetorheological fluid dynamometer bench as shown in Fig. 1, viscosity of magnetorheological Fluid increases dramatically under the action of external magnetic field and imposes resistance moment on the moving object and we 
can get the value of the torque, namely output torque of the rotating parts, based on excitation coil size and structure size, rotating speed based on speed sensor, so that we can get the output power of the rotating parts by the formula (1).

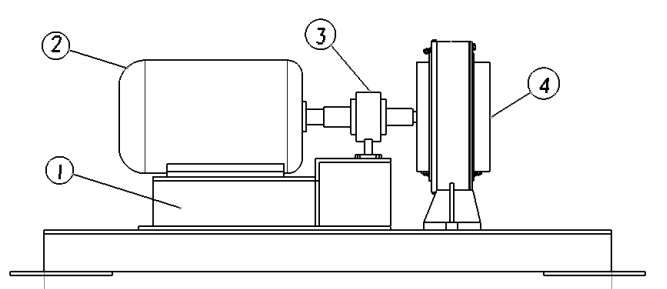

1. chassis ; 2.rotating parts ; 3. speed sensor ; 4. MRF dynamometer

Fig 1. Magnetorheological fluid dynamometer test bench.

\section{Torque Calculation}

Currently, the most common view about the constitutive nature of MRF is to treat magnetorheological fluid as a Bingham fluid. The constitutive equation[9] is:

$$
\tau=\tau_{B}+\eta \gamma
$$

Where $\tau$ is the shear stress $\left(\mathrm{P}_{\mathrm{a}}\right) ; \tau_{B}$ is the dynamic yield stress $\left(\mathrm{P}_{\mathrm{a}}\right), \eta$ is the liquid viscosity $\left(\mathrm{P}_{\mathrm{a}} \cdot \mathrm{s}\right) ; \gamma$ is the shear rate[10].

$$
\gamma=\omega \cdot \frac{r}{h}
$$

Where $\omega$ is the MRF angular velocity at any position $\left(\mathrm{rad} \cdot \mathrm{s}^{-1}\right), \mathrm{r}$ and $\mathrm{h}$ are radius and thickness of disk.

Through the study of magneto-rheological fluid existing products, we chose disk magnetorheological fluid power absorption device, torque analysis as shown in Fig. 2

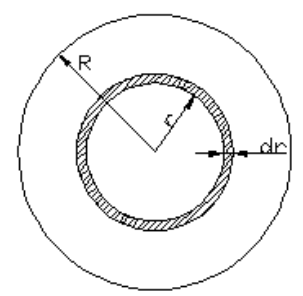

(a)

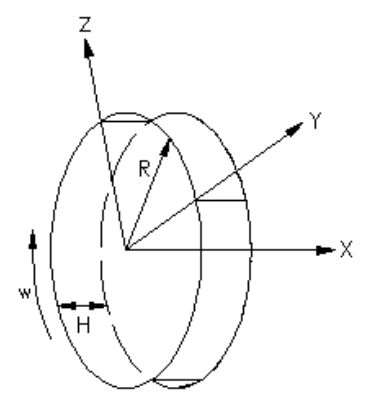

(b)

Fig 2. Magnetorheological Fluid Torque Analytical Diagram.

The shaft rotates around $x$-axis in angular velocity $(\omega)$, shears with MRF that contacts the disk and forms a disc shape by the magnetorheological fluid that has been through magnetorheological effect. Assumed the outer diameter of disc shape is $\mathrm{R}$, we take the micro annular disc-shaped magnetorheological fluid whose width is $d_{r}$ to study. At the position of radius $r$, the micro torque of micro annular disc-shaped magnetorheological fluid is:

$$
d_{T}=r d_{F r}
$$

Where $\mathrm{r}$ is the radius of micro disk; $d_{F r}$ is the micro shear stress, in addition

$$
\begin{aligned}
& d_{F r}=\tau d_{s} \\
& d_{s}=2 \pi r d_{r}
\end{aligned}
$$

We can get load torque that single disk can offer from formula (2) (6)

$$
T=\frac{2}{3} \pi \tau_{B} R^{3}+\frac{1}{2 h} \pi \eta \omega R^{4}
$$

As is shown in formula (7),load torque consists of two parts. First part relates to magnetic induction intensity and structure size $T_{B}=\frac{2}{3} \pi \tau_{B} R^{3}$ and second part is viscous resistance moment of magnetorheological fluid $T_{\eta}=\frac{1}{2 h} \pi \eta \omega R^{4}$. Total load torque equals the sum of two parts: $T=T_{B}+T_{\eta}$. Volume of magnetorheological fluid:

$$
V=\pi R^{2} H
$$

From formula (7) and (8), we can get:

$$
\frac{H}{R}=\frac{3}{4} \cdot \frac{T_{B}}{T_{\tau}} \cdot \frac{\eta \omega}{\tau_{B}}
$$

Formula shows that geometric constraints of disk magnetorheological fluid power measurement device relates to the following parameters: the material properties of magnetorheological fluid $\left(\eta / \tau_{B}\right)$, torque component ratio expected value $\left(T_{B} / T_{\tau}\right)$, input rotational speed $\omega$.

\section{Magnetic Field Analysis}

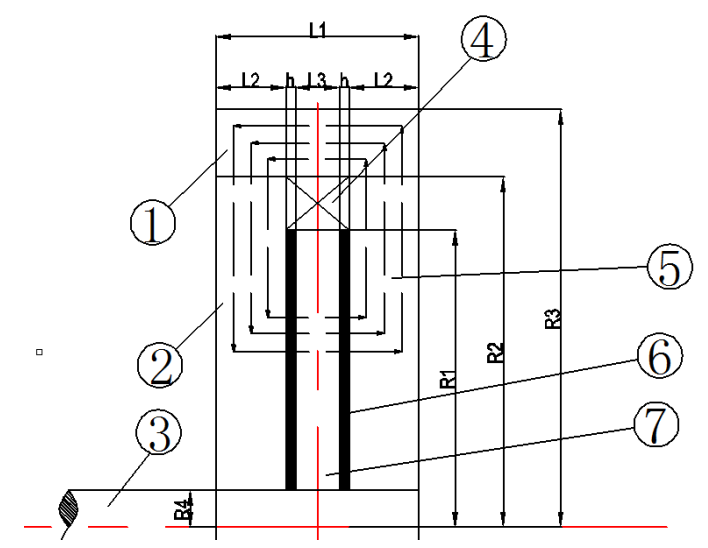

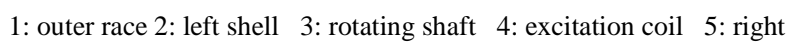
shell 6: magnetorheological fluid, 7: turntable

Fig 3. Double disk type magnetorheological fluid magnetic circuit diagram. 
Double disk type magnetorheological fluid magnetic circuit diagram as shown in Fig. 3, the rotating shaft drives the rotation of the turntable. There is disk type MRF on both sides of turntable respectively, with thickness of $h$. When the turntable rotates, the excitation coil is energized, and imposes magnetic field on magnetorheological fluid and magnetorheological Fluid produces magnetorheological effect thus providing the load torque. The dotted line with an arrow in the Fig. is the trend of virtual magnetic induction line: outer race $\rightarrow$ left shell $\rightarrow$ magnetorheological fluid $\rightarrow$ turntable $\rightarrow$ magnetorheological fluid $\rightarrow$ right shell $\rightarrow$ outer race forms a loop. Thus each disk needs to provide the load torque of half shaft torque, namely:

$$
\frac{T}{2}=\frac{2}{3} \pi \tau_{B} R^{3}+\frac{1}{2 h} \pi \eta \omega R^{4}
$$

Reluctance calculation is similar to the calculation of the resistance, so it is also called the magnetic Ohm 's Law[11]. Reluctance calculation formula:

$$
R_{M}=\frac{L}{\mu \cdot s}=\frac{L}{\mu_{R} \mu_{0} s}
$$

Where $\mathrm{L}$ is the circuit length, $\mu$ is the material permeability, $\mathrm{s}$ is the magnetic circuit cross section, $\mu_{R}$ is the material relative magnetic permeability, $\mu_{0}$ is the permeability of vacuum, $\mu_{0}=4 \pi \times 10^{-7} \mathrm{~N} \cdot A^{-2}$.

As is shown in formula (11), reluctance value is proportional to magnetic circuit length and inversely proportional to magnetic permeability and magnetic circuit cross section. Reluctance value can be changed by these three parameters in the design. Fig. 3 loop is series arrangement.

When magnetic circuit passes turntable 7, magnetorheological fluid 6 ,and outer race 1 ,substituted formula (11) and get:

$$
\begin{gathered}
R_{M 7}=\frac{L_{3}}{\pi\left(R_{1}^{2}-R_{4}^{2}\right) \mu_{7}} \\
R_{M 6}=\frac{h}{\pi\left(R_{1}^{2}-R_{4}^{2}\right) \mu_{6}} \times 2 \\
R_{M 1}=\frac{L_{1}-L_{2}}{\pi\left(R_{3}^{2}-R_{2}^{2}\right) \mu_{1}}
\end{gathered}
$$

When magnetic circuit passes left and right shell, because they are symmetrical, substituted formula (11) and get:

$$
R_{M 2}=\frac{\ln \left(\frac{R_{2}+R_{3}}{2}\right)-\ln R_{4}}{2 \pi L_{2} \mu_{2}}
$$

$$
R_{M 5}=R_{M 2}
$$

So the total reluctance of reluctance loop is:

$$
R_{M}=R_{M 1}+R_{M 2}+R_{M 5}+R_{M 6}+R_{M 7}
$$

According to the Ampere circuital theorem in magnetic circuit[12], magnetomotive force of magnetic circuit:

$$
V_{m}=\Phi R_{M}=N I
$$

Here $\Phi$ is the magnetic flux, $\mathrm{N}$ is number of coil; I is the coil current.

When determining the material and structure parameters of the magnetic circuit, the total reluctance and action area under the corresponding magnetic induction intensity of magnetic circuit are uniquely determined, load torque and magnetic induction intensity exist one-to-one relationships.

\section{Determination of Current I}

After getting the magnetomotive force of magnetic circuit, we need a further determination of specific parameters of magnetic excitation coil, mainly including coil conductor diameter (coil current) and turns per coil. Magnetic excitation coil conductor diameter is determined by the formula[13]:

$$
d=\sqrt{\frac{4 \rho_{c} d_{c p} N I}{U}}
$$

Where $\rho_{c}$ is the resistivity of the wire material $\left(\Omega \cdot \mathrm{mm}^{2} \bullet \mathrm{m}^{-1}\right), \mathrm{d}_{\mathrm{cp}}$ is the coil average diameter $(\mathrm{m}), \mathrm{NI}$ is the coil ampere turns, $\mathrm{U}$ is the supply voltage.

\section{Motor Dynamometer Device Design}

Take QABP-160M2A motor as example. Parameters are as follows:

Table 1. QABP-160M2A technical data.

\begin{tabular}{cccc}
\hline $\begin{array}{c}\text { Rated Power } \\
\mathbf{P}(\mathbf{k W})\end{array}$ & $\begin{array}{c}\text { Rated } \\
\text { Torque } \\
\mathbf{T}(\mathbf{N} \cdot \mathbf{m})\end{array}$ & $\begin{array}{c}\text { Rated Rotational } \\
\text { Speed } \\
\mathbf{n}\left(\mathbf{r} \cdot \mathbf{m i n}^{-\mathbf{1}}\right)\end{array}$ & $\begin{array}{c}\text { Max } \\
\text { Torque/Rated } \\
\text { Torque(times) }\end{array}$ \\
\hline 11 & 35 & 2930 & 3 \\
\hline
\end{tabular}

We choose Tider MFR270/50 type magnetorheological fluid, whose technical data is as table 2. The constitutive equation is[14]:

$$
\tau_{B}=64.72 \times[1-\exp (-1.63 B)]
$$

Table 2. Magnetorheological Fluid Technical Data.

\begin{tabular}{cccc}
\hline $\begin{array}{c}\text { Magneto- } \\
\text { rheological } \\
\text { Fluid Type }\end{array}$ & $\begin{array}{c}\text { Relative } \\
\text { Permeability }\end{array}$ & $\begin{array}{c}\text { Viscosity at } \\
\mathbf{2 0}^{\mathbf{0}} \mathbf{C}(\mathbf{c P})\end{array}$ & $\begin{array}{c}\text { Magnetic } \\
\text { Saturation } \\
\text { Intensity } \\
(\mathbf{T})\end{array}$ \\
\hline $\begin{array}{c}\text { Tider } \\
\text { MFR270/50 }\end{array}$ & 8 & 0.27 & $1 \sim 2$ \\
\hline
\end{tabular}


Permeability material used for the magnetic structure(including left and right shell, outer race and turntable) around coil is $\mathrm{A}_{3}$ steel whose relative permeability can increase from the initial several hundreds to thousands under different magnetic induction intensity, but it has no change in magnitude, so we ignore the change and choose relative permeability $\mu_{r A}=800$, magnetic saturation intensity $\mathrm{B}_{\mathrm{A}}=2.14 \mathrm{~T}[15]$.

Structure of magnetorheologicalfluid dynamometer is shown in Fig. 4.

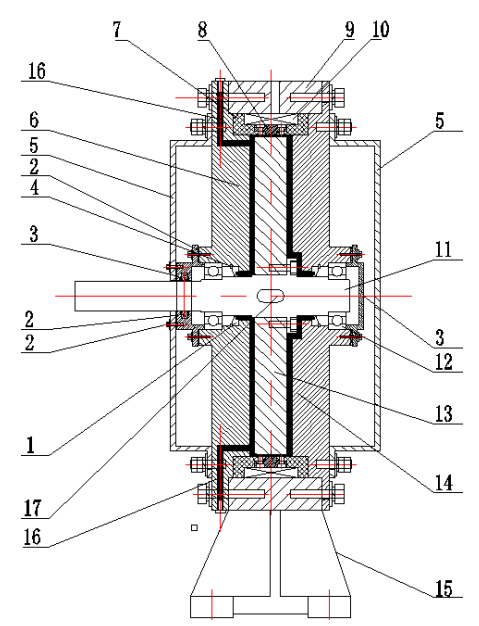

1: framework oil seals; 2 : felt ring seal;3 bearing end cover;4: clip ring for hole;5:cooling water-jacket;6:left shell;7:coil support ;8:isolation magnetic ring;9:outer race;10:coil;11:rotatingshaft;12:bearing;13:turntable;14:right shell;15:base;16: magnetorheological fluid injection hole;17: A type key

Fig 4. Magnetorheological Fluid Dynamometer.

Considering structure size against Fig. 3, magnetorheological fluid effective outer diameter is $\mathrm{R}_{1}=0.1 \mathrm{~m}$, coil outer diameter $\mathrm{R}_{2}=0.11 \mathrm{~m}$,rotating shaft diameter $R_{4}=0.015 \mathrm{~m}$. To make full use of magnetorheological fluid, magnetorheological fluid should saturate first and from table 2, we chose magnetic saturation intensity limit $\mathrm{B}_{\mathrm{M}}=1 \mathrm{~T}$, so the when magnetorheological fluid saturates, the magnetic flux is $\Phi=3.07 \times 10^{-2} \mathrm{~Wb}$. Because magnetic flux in every magnetic circuit loop is same, substitute data and get: $R_{3}=0.13 \mathrm{~m}$, $\mathrm{L}_{2}=0.022 \mathrm{~m}$.

As for the turntable, because its section is the same as magnetorheological fluid and magnetic saturation intensity is bigger than magnetorheological fluid's, its thickness is ok if slightly bigger than magnetorheological fluid's. We chose magnetorheological fluid thickness $\mathrm{h}=0.0015 \mathrm{~m}[16]$ and considering the strength requirement of turntable, its thickness chooses $\mathrm{L}_{3}=0.008$ mand substituted formula (12) (16), we get reluctance of each part: $\mathrm{R}_{\mathrm{M} 7}=259 \mathrm{H}$ 1, $\quad \mathrm{R}_{\mathrm{M} 6}=9728 \mathrm{H}^{-1}, \quad \mathrm{R}_{\mathrm{M} 2}=\mathrm{R}_{\mathrm{M} 5}=31565 \mathrm{H}^{-1}, \quad \mathrm{R}_{\mathrm{M} 1}=2179 \mathrm{H}^{-1}$. Therefore, the total reluctance is: $\mathrm{R}_{\mathrm{M}}=75296 \mathrm{H}^{-1}$.

When the dynamometer works, the torque increases as the current increases, so the coil should have the ability to provide maximum torque current. From table 1, we know $\mathrm{T}_{\max }=105 \mathrm{~N} \cdot$ mand the rotation speed is $\mathrm{n}=1000 \mathrm{r} \cdot \mathrm{min}^{-1}$, namely $\omega=105 \mathrm{rad} \cdot \mathrm{s}^{-1}$. According to formula (10)、 and (18),we can get: dynamic yield stress $\tau_{B}=21.96 \times 10^{3} p_{a}$, magnetic induction $\mathrm{B}=0.25 \mathrm{~T}$, magnetomotive force $\mathrm{V}_{\mathrm{m}}=577.78 \mathrm{~A}$.

Coil wire is copper enameled wire with good conductivity, whose standard specification as table 3 . Voltage is common voltage $U=12$ V. From (18) and (19), we know conductor diameter $\mathrm{d}=0.865 \mathrm{~mm}$. According to the experience of each square millimeter of wire can pass through the $5 \mathrm{~A}$ current, namely coil current $\mathrm{I}=2.9 \mathrm{~A}$. From (18), we get coil turns: $\mathrm{N}=199$, the coil resistance $R_{\text {coil }}=4.19 \Omega$.

Thus, we finish the calculation process of motor power $\rightarrow$ rotational speed $\rightarrow$ torque $\rightarrow$ current. As for the device structure size that has been known, if rotational speed measured by speed sensors, the power given by the output current, one-to-one correspondence between current and torque, by calculating the corresponding load torque calculated from current, we can get power motor of rotating parts.

Table 3.Copper Enameled Wire Specification.

\begin{tabular}{ccccc}
\hline $\begin{array}{c}\text { Copper } \\
\text { Core } \\
\text { Nominal } \\
\begin{array}{c}\text { Diameter } \\
(\mathbf{m m})\end{array}\end{array}$ & $\begin{array}{c}\text { Copper } \\
\text { Enameled } \\
\text { Wire Max } \\
\text { Diameter } \\
(\mathbf{m m})\end{array}$ & $\begin{array}{c}\text { Copper } \\
\text { Core Cross } \\
\text { Section } \\
\left(\mathbf{m m}^{2}\right)\end{array}$ & $\begin{array}{c}\text { No. } \\
(\mathbf{c m})\end{array}$ & $\begin{array}{c}\text { Resistivity } \\
\left(\Omega \cdot \mathrm{mm}^{2} \cdot \mathrm{m}^{-1}\right)\end{array}$ \\
\hline 0.83 & 0.89 & 0.5411 & 11.2 & \\
0.86 & 0.92 & 0.5809 & 10.9 & 0.01851 \\
0.9 & 0.96 & 0.6362 & 10.4 & \\
\hline
\end{tabular}

Both engine and motor rotating parts have the character of decreasing and increasing torque, namely torque will change when rotational speed changes. As for the above device, we can increase or decrease the current to control the load torque, and further control the rotational speed of rotating parts.

\section{Conclusion}

(1) Put forward a new theoretical dynamometer design method, the calculation process of torque and reluctance during the design of magnetorheological fluid dynamometer and a determination method of current, turns, diameter and other parameters.

(2) Taking some motor as example into practice proves its feasibility of this method, enlarges dynamometer range and provides a theoretical basis for the design of magnetorheological fluid dynamometer.

(3) Magnetorheological fluid dynamometer design process still ha some problems, such as sealing, cooling and control problems. If these problems are solved, the design of the dynamometer will be more perfect.

\section{Acknowledgements}

This research was supported by the modern automobile service engineering subject platform construction in Shanghai City (A-0507-13-0226). 
The first author Luo Yiping was born in 1966. He gained a master's degree of Industrial Engineering in Shanghai Jiao Tong University in 1987. Now he is a professor in Shanghai University of Engineering Science.

The second author Xu Biao was born in septemper.1990 in Anhui province. He got bachelor's degree of vehicle engineering in Anhui Polytechnic University in 2012 and now is a graduate student in Shanghai University of Engineering Science.

The third author Ren Hongjuan was born in 1978 in Shandong province. She gained a master's degree of Power Machinery and engineering in Shandong University in 2002. Now she is an associate professor in Shanghai University of Engineering Science.

\section{References}

[1] Meng Li, Jiangang Lv, Yong Wei. Study on the System of Tracked Vehicles Magnetorheological Fluid Damper Suspension[J]. Mechanical Design, 2004, 21(12): 52-55.

[2] Yanrong Yang, Huiyong Shan, Yong Wei. The Theoretical Analysis and Design of a Cylindrical Magnetorheological Brake [J]. Electromechanical Engineering Technology , 2005, 34(10): 15-16.

[3] Lin Zhang. Research and Design of Disk Type Magnetorheological Transmission Mechanism [J]. Mechanical Design, 2009, 26(1): 31-32.

[4] Bossis G, Mathis C, Mimouni Z. Magneto rheolgical suspensions[J]. Euro phys Lett, 1990, 11(2): 133-137.

[5] Kormann C, Laun H. M, Richter H J. MR Fluid with NanoSized Magnetic Particles Technology [J]. International Journal of Modern Physics, 1996, 10(23): 3167-3172.

[6] Cutillas S. Bossis G CebersA [J]. Vhys Rev E, 1998, 57(1):
804-811.

[7] Gopalswamy S, Linzell S M, Jones G L. MR fluid clutch with minimized reluctance $[\mathrm{P}]$. USA:US Patent: 5896965,1999 .

[8] Jianhua Ni, Zhiqian Zhang, Ke Zhang. A New Type of Magnetorheological Damper and Its Application in Semiactive Control of Vehicle Suspension [J]. Mechanical Science and Technology , 2004, 23(1): 4-6.

[9] O. Ashour, A. Craig. Magnetorheological Fluid: materials, characterization, and devices [J]. Int. Mater. Syst. struct, 1996, 7(2): 123-130.

[10] Jian Chang, Yunmin Yang, Xianghe Peng. Research on testing device for magnetorheological fluid properties [J]. Chinese Journal of scientific instrument, 2001, 22(4): 354358.

[11] Wu Ai, Cheng Li. Circuit and Magnetic Circuit [M]. Wuhan City: Huazhong University of Science and Technology press, 2002.

[12] Canbin Liang, Guangrong Qin, Zhujiang Liang. Electromagnetics $[\mathrm{M}]$. Beijing: Higher Education Press, 1980.

[13] Jinming Tian. Design and Application of Electromagnetic Clutch [M]. Suzhou: Jiangsu Science and Technology Publishing House, 1982.

[14] Kejun Jiang, Chengye Liu. Design and Performance Simulation of Magnetorheological Fluid Clutch [J]. Computer Sumulation, 2011, 28(8): 337-341.

[15] Jun Zheng. Magnetorheological Transmission Theory and Experimental Study [D]. Chongqing: Chongqing University, 2008.

[16] W. H. Li and, H. Du. Design and Experimental Evaluation of a Magnetorheological Brake [J]. The International Journal of Advanced Manufacturing Technology, 2003, 21(7): 508-515. 